\title{
POPULATION SYNTHESIS MODELS
}

\author{
GUSTAVO BRUZUAL A.
}

C.I.D.A.

Apartado 264, Mérida 5101-A, Venezuela

\section{Introduction}

Increasingly complex population synthesis models have been developed during the last few years. Among the different authors working in the field, these groups have been particularly active: Arimoto \& Yoshii (1987), Guiderdoni \& Rocca-Volmerange (1987), Buzzoni (1989), Fritze-v. Alvensleben \& Gerhard (1994), Bressan, Chiosi, \& Fagotto (1994), Bruzual \& Charlot $(1993,1995)$. The basic astrophysical ingredients used in these models are the stellar evolutionary tracks and the stellar spectral libraries (either empirical or theoretical). The computational algorithms are different in each set of models, but, one way or the other, these models depend on the same adjustable parametric functions: (1) the stellar initial mass function (IMF); (2) the star formation rate (SFR); and (3) the rate of chemical enrichment (in some models $Z=Z_{\odot}=$ constant). Most of the recent codes use the isochrone synthesis algorithm, which allows to compute the evolution of a simple stellar population (SSP), and from it, by a convolution integral, the properties of more complex composite stellar populations (CSP).

From the population synthesis models one obtains basically the time evolution of the galaxy spectral energy distribution (SED), integrated colors, and spectral indices. From the model SEDs one can derive a spectral age for a given problem galaxy SED (i.e. the age of the model that reproduces best the problem SED). The spectral age depends, in general, on the IMF, the SFR, the assumed metallicity, and the particular set of models used. From the selected best fitting model and assuming a cosmological model, one can relate galaxy magnitude and colors with redshift $(z)$, or galaxy number counts with galaxy apparent magnitude, etc. All these topics will be discussed in detail during this meeting. 
Are population synthesis models consistent? A desirable property of population synthesis models is that all the models produce the same answer for the same set of input parameters. This is in fact not always the case. Some of the discrepancies among the models may be expected from the differences in the ingredients used to build them, but one should understand these differences and their origin. To investigate the origin of the remaining differences, one can fix a subset of the input parameters and vary the remaining part.

\section{Comparison of 3 Sets of Models}

A detailed comparison of the differences in the predictions from the models by Bruzual \& Charlot (1995, hereafter BC95), Worthey (1994, hereafter W94), and Bertelli, Bressan, Chiosi, Fagotto, \& Nasi (1994, hereafter BBCFN) has been performed by Charlot, Worthey \& Bressan (1996, hereafter CWB96). I will comment here on the main conclusions of this work.

The BC95 models constitute a revised, updated, and debugged version of the Bruzual \& Charlot (1993, hereafter BC93) models. Evolutionary tracks from the Geneva (Schaller et al. 1992, Charbonnel et al. 1995) and Padova (Bressan et al. 1993) groups for solar metallicity have been used to construct two parallel sets of models. The spectral library is mostly empirical, based on the Gunn \& Stryker (1983) atlas, and improves the coverage of the coolest giants in the optical range with the observations by Fluks et al. (1994). The color vs. $T_{\text {eff }}$ and bolometric correction (BC) vs. $T_{\text {eff }}$ relationships used to transform the theoretical HR diagram to the observational plane have been revised to follow as close as possible the standard calibrations.

The W94 models are based on the evolutionary tracks by VandenBerg (1985) and the revised Yale isochrones (Green, Demarque, \& King 1987). The stellar SEDs are obtained from the Kurucz (1992) set of model atmospheres for $T_{\text {eff }}>3750 \mathrm{~K}$, and Bessel et al. (1991, and references therein) for cooler stars (supplemented by optical SEDs from the Gunn \& Stryker atlas). These models cover a wide range of metallicities.

The BBCFN models are based on evolutionary tracks computed by Alongi et al. (1993), Bressan et al. (1993), and Fagotto et al. (1994). The Kurucz, (1992) set of model atmospheres is used for stars of $T_{\text {eff }}>3500$ $\mathrm{K}$, and observed spectra for cooler stars. These models as well cover a wide range of metallicities.

The BC95, W94, and BBCFN sets of models sample and combine a good fraction of the theoretical and empirical data available in the literature. By computing the same galaxy model with the 3 codes one can explore the sensitivity of the population synthesis models to changes in the input in- 


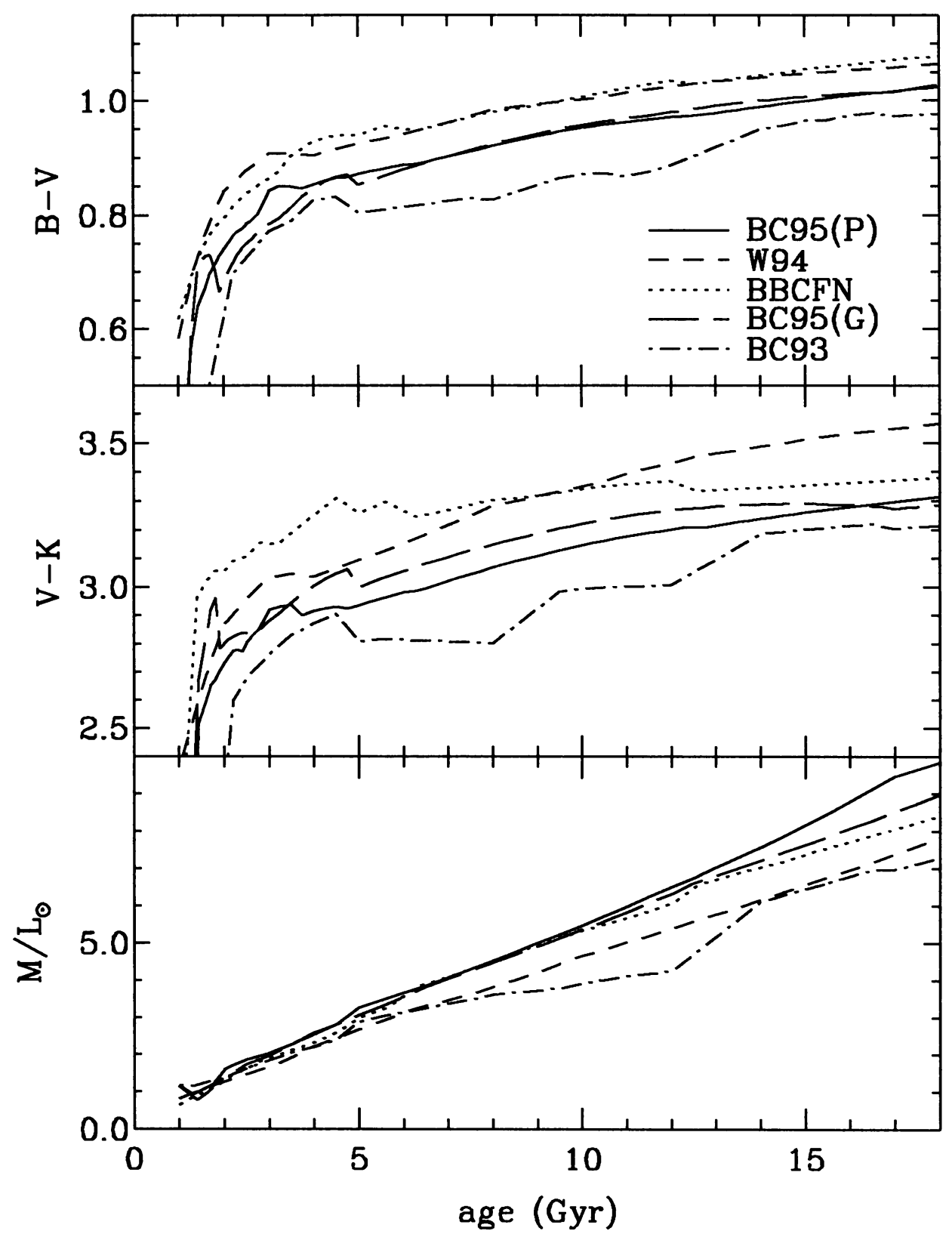

Figure 1. Evolution in time of the $B-V$, and $V-K$ colors, and the $M / L_{V}$ ratio for the 3 sets of models discussed in the text. 
gredients. CWB96 chose to compute a solar metallicity SSP for the Salpeter (1955) IMF $\left(m_{I}=0.15, m_{U}=2 M_{\odot}\right)$ in the age range from 1 to $17 \mathrm{Gyr}$. This population can be computed with the current versions of the 3 sets of models.

Figure 1 shows the evolution in time of the $B-V$ and $V-K$ colors, and the $M / L_{V}$ ratio predicted by the 3 sets of models for this SSP. For the BC95 models the results are shown for the Padova group evolutionary tracks (P) as well as for the Geneva group tracks (G). For comparison, the prediction of the BC93 models is also included. From this figure it is apparent that at late ages the W94 and BBCFN models are 0.05 mag redder in $B-V$ than the $\mathrm{BC} 95$ models (either $\mathrm{C}$ or $\mathrm{M}$ ), which in turn are about 0.06 mag redder than the BC93 model. In $V-K$ the differences are more dramatic. The W94 model is more than 0.2 mag redder than the BC95 models, which are only 0.04 mag below the BBCFN model. The BC93 model is again the bluest. The $M / L_{V}$ ratios are in good agreement for the BC95 and the BBCFN models up to 15 Gyr. The W94 and BC93 models predict considerably lower values of this ratio.

By using the BC95 empirical spectral library with the three evolutionary codes, CWB96 explore to what extent the indicated color differences reflect the different prescriptions about stellar evolution assumed in each code. CWB96 conclude that: (1) The bluer evolution in $B-V$ of the BC95 with respect to the W94 and BBCFN models cannot be attributed to differences in stellar evolution. It must come from differences in the spectral calibrations. (2) Much of the discrepancy in the evolution of $V-K$ does appear to result from the different stellar evolutionary tracks used. (3) Differences in stellar evolution account for nearly all of the differences in $M / L_{V}$ in the 3 models. W94 models are redder in $V-K$ because of a larger number of upper RGB stars in Worthey's isochrones than in the BC95 models. The difference between BBCFN and BC95 in $V-K$ is due to a different treatment of the TP-AGB evolutionary phase.

To study the effects on the population models of changing the spectral calibrations, CWB96 compute SSP models with the 3 codes using the Geneva group evolutionary tracks in all of them, but keeping the original spectral calibration used in the models. Their conclusions are as follow: (1) Dwarf stars are $0.05 \mathrm{mag}$ redder in W94 and BBCFN than in BC95 for $\log T_{\text {eff }}>$ 3.6. (2) Cooler dwarfs are about 0.1 mag redder in $B-V$ in W94 and BC95 than in BBCFN. These cool dwarfs contribute very little to the total SED. (3) For giants with $\log T_{\text {eff }}>3.65, B-V$ is $0.06 \mathrm{mag}$ redder in W94 and BBCFN than in BC95 or the Johnson (1966) calibration. CWB96 think that this difference is introduced by the Kurucz (1992) model atmospheres, which are redder than the observed SEDs. (4) BC95 use the $V-K$ vs. $T_{\text {eff }}$ calibration of Ridgway et al. (1980). W94 stars are 1 


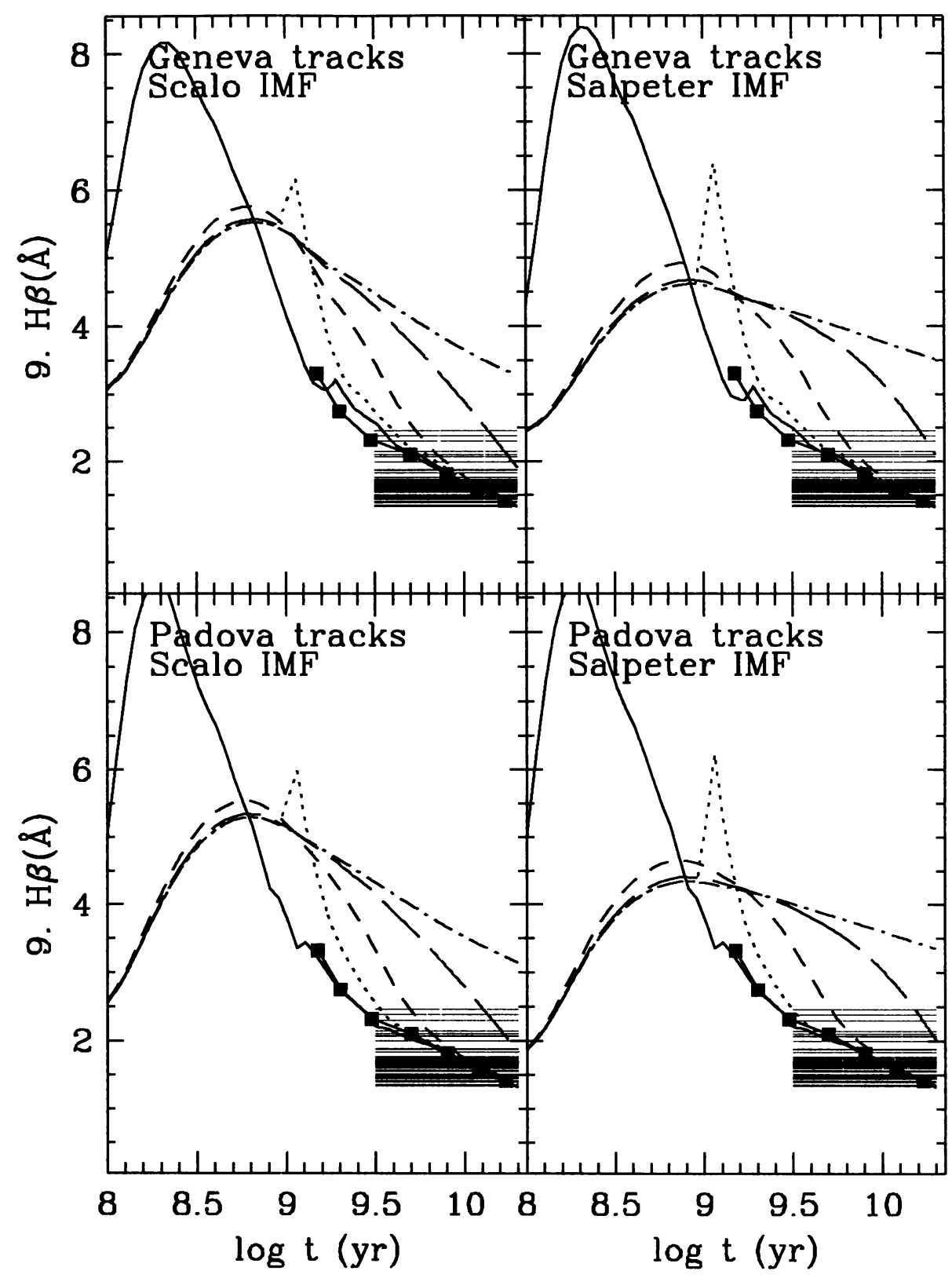

Figure 2. Evolution in time of the $\mathrm{H} \beta$ spectral index. The meaning of the different lines and symbols is explained in the text. 
mag redder than this calibration cooler than $\log T_{\text {eff }}=3.51$. BBCFN stars are in turn $1 \mathrm{mag}$ bluer than the Ridgway et al. calibration. (5) Cool giants $(3300 \mathrm{~K})$ are about $1 \mathrm{mag}$ brighter at $V$ (for same bolometric luminosity) in the BBCFN model than in the BC95 and W94 models. The BC used by BBCFN are more positive than the ones used in the other models, which follow more closely the standard calibration.

CWB96 conclude that despite the large differences in the photometric calibrations, color evolution is very similar in the 3 models. The large differences in the adopted BC's and $T_{\text {eff }}$ scale for giants in the BC95 and BBCFN models conspire to produce an almost identical $V-K$ evolution in these two cases. W94 giants are not as bright in $V$ and $V-K$ is redder. However, this is misleading agreement. Differences in stellar evolution prescriptions can produce differences of up to 0.2 in $V-K$ and of 15 to $20 \%$ in $M / L_{V}$.

\section{Spectral Indices and Galaxy Ages}

BC95 have computed the 21 spectral indices introduced by Worthey (1992) using the fitting functions coefficients listed in Worthey et al. (1994). The pseudo-continuum flux levels have been determined from the spectrum assigned to each star in the BC95 models. BC95 have explored the sensitivity of the indices to the IMF and the SFR, and have compared their results with those obtained by Worthey et al. Figure 2 shows the behavior in time of the $\mathrm{H} \beta$ spectral index for several models. The evolutionary tracks and the IMF are indicated inside each panel. The solid line corresponds to an SSP. The dotted line represents a burst of star formation lasting for $1 \mathrm{Gyr}$. The SFR is zero afterwards. The dashed and long-dashed lines represent the behavior of models with exponentially decaying SFR with e-folding time $\tau=1$, and $5 \mathrm{Gyr}$, respectively. The dotted-dashed lines corresponds to a constant SFR model. The horizontal lines drawn from $\log t=9.5$ to 10.3 indicate the value of the index measured by González (1993) in his E galaxy sample. The length of these lines is arbitrary. The solid dots represent the values of the index derived by Worthey (1992) for the $Z=Z_{\odot}$ SSP with the Salpeter IMF. There is excellent agreement of the BC95 values with Worthey (1992). Figure 3 shows the behavior of the Mgb index for the same models.

Figures 2 and 3 show clearly how the spectral indices depend on the SFR, and to a lesser amount on the IMF and stellar physics. Even though some of the $\mathrm{E}$ galaxies in González sample have values of the indices reached at by an SSP at ages around $3 \mathrm{Gyr}$, the same values are reached at later ages by mostly old populations in which star formation has been going on over a longer time span. Galaxy ages $>10 \mathrm{Gyr}$ do not seem to be ruled out 


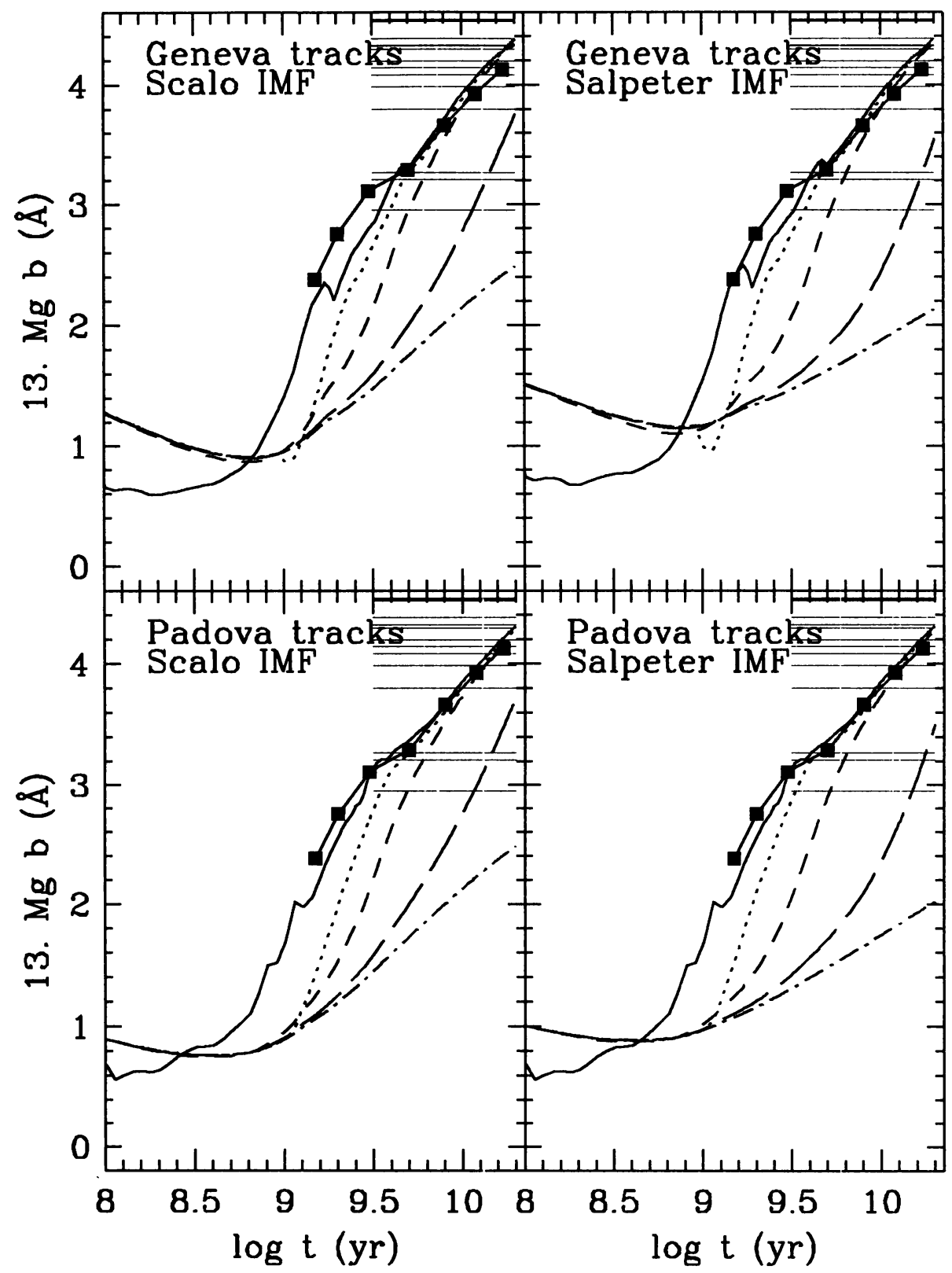

Figure 3. Evolution in time of the Mgb spectral index. The meaning of the different lines and symbols is explained in the text. 
by the observations. Young galaxy ages based on fits to the values of the indices predicted for SSP's are uncertain as much as our knowledge of the history of star formation in these systems.

\section{Conclusions}

Users of population synthesis models should be aware of the sensitivity of their favorite models to the physics and assumptions that went into the models. We, model builders, should pay more attention to the stellar calibrations, spectral libraries, evolutionary tracks and their physics, and not only to writing more sophisticated algorithms. Observers should make an effort to improve the spectral calibrations in those regions of the HR diagram with the highest uncertainties, which frequently dominate the behavior of integrated populations. Some E/S0 galaxies may have a spectral (or index) age as low as 8-10 Gyr, but much younger ages (3-5 Gyr) may not be required if the history of star formation in the galaxy is properly taken into account.

\section{References}

Alongi, M., Bertelli, G., Bressan, A., Chiosi, C., Fagotto, F., Greggio, L. \& Nasi, E. 1993, A\&AS, 97, 851

Arimoto, N., \& Yoshii, Y. 1987, A\&A, 173, 23

Bertelli, G., Bressan, A., Chiosi, C., Fagotto, F., \& Nasi, E. 1994, A\&AS, 106, 275 (BBCFN)

Bessel, M.S., Brett, J.M., Scholz, M., \& Wood, P.R. 1991, A\&AS, 89, 335

Bressan, A., Fagotto, F., Bertelli, G., \& Chiosi, C. 1993, A\&AS, 100, 647

Bressan, A., Chiosi, C., \& Fagotto, F. 1994, Ap.JS, 94, 63

Bruzual A., G., \& Charlot, S. 1993, Ap.J, 405, 538 (BC93)

Bruzual A., G., \& Charlot, S. 1995, (in preparation, BC95)

Buzzoni, A. 1989, Ap.JS, 71, 817

Charbonnel, C., Meynet, G., Maeder, A., Schaller, G., \& Schärer, D. 1995, A\&AS, submitted

Charlot, S., Worthey, G. \& Bressan, A. 1996, Ap.J, Feb 1, (in press, CWB96).

Fagotto, F., Bressan, A., Bertelli, G., \& Chiosi, C. 1994, A\&AS, 105, 29

Fluks, M.A., Plez, B., Thé, P.S., de Winter, D., Westerlund, B.E., \& Steenman, H.C. 1994, A\&AS, 105, 311

Fritze-von Alvensleben, U.A. \& Gerhard, O.E. 1994, A\&A, 285, 751

González, J.J. 1993, PhD Thesis, University of California, Santa Cruz

Green, E.M., Demarque, P. \& King, C.R. 1987, The Revised Yale Isochrones and Luminosity Functions (New Haven: Yale University Observatory)

Guiderdoni, B. \& Rocca-Volmerange, B. 1987, A\&A, 186, 1

Gunn, J.E. \& Stryker, L.L. 1983, Ap.JS, 52, 121

Johnson, H.L. 1966, AR.AA, 4, 193

Kurucz, R.L. 1992, in The Stellar Populations of Galaxies, IAU Symp. 149, eds. B. Barbuy \& A. Renzini (Dordrecht: Kluwer), 225

Ridgway, S.T., Joyce, R.R.., White, N.M., \& Wing, R.F. 1980, Ap.J, 235, 126

Salpeter, E.E. 1955, ApJ, 121, 161

Schaller, G., Schärer, Meynet, G., \& Maeder, A. 1992, A\&AS, 96, 269 
VandenBerg, D.A. 1985, Ap.JS, 58, 711

Worthey, G. 1992, PhD Thesis, University of California, Santa Cruz

Worthey, G. 1994, Ap.JS, 95, 107 (W94),

Worthey, G., Faber, S.M., González, J.J., \& Burstein, D. 1994, Ap.JS, 94, 687

\section{DISCUSSION:}

Peletier: The discrepancy you showed between your Mgb index and Worthey's might well be related to the way you have measured the indices. The way indices are measured on the Lick system is very hard to reproduce, since spectra of individual stars have to be transformed to a certain resolution and continuum shape. It might well be worth recalibrating the indices on flux calibrated spectra for all the stars of the Worthey et al. paper.

Bruzual: Your comment is well taken. However, since the oral version of this talk, G. Worthey pointed out to me that the fitting function coefficients in the copy of his thesis that I have are in error, and that I should use the ones in Worthey et al. (1994). After doing this, the discrepancies that I mentioned become less significant. But I still think that it may be useful to recalibrate the indices.

Bender: You showed that colors and line strengths can differ considerably for different stellar population models. What about the slopes? Can, e.g., relative ages be determined more accurately than absolute ages from measurements of $\mathrm{Mgb}$ or $\mathrm{H} \beta$ ?

Bruzual: The slopes are considerably more stable than the intercepts. From the figures presented here you can see that it is possible to guess how much younger (or older) a stellar population is than a fiducial age. Even for different SFR's and IMF's the slope of the indices vs. age lines (at large ages) are not far from one another.

Worthey: Are the short timescale bumps and wiggles seen in all recent population synthesis models real, or can we smooth them away?

Bruzual: I think they are produced by numerical noise and that they can be smoothed out.

Renzini: Those little wiggles are just due to the numerics, i.e. to the specific numerical algorithm used in the synthesis. They are not real.

Renzini: All the comparisons you have shown refer to solar metallicity. Yet, the situation may be even worse for $Z>Z_{\odot}$, as theoretical model atmospheres become more and more unreliable, and empirical spectral libraries more and more incomplete. Do you agree with this statement?

Bruzual: I could not agree more with your comment. In fact these uncertainties have prevented S. Charlot and myself from extending our population synthesis models to metallicities above solar. The below solar regime is less uncertain and population synthesis for $Z \leq Z_{\odot}$ is more reliable than for $Z>Z_{\odot}$. 


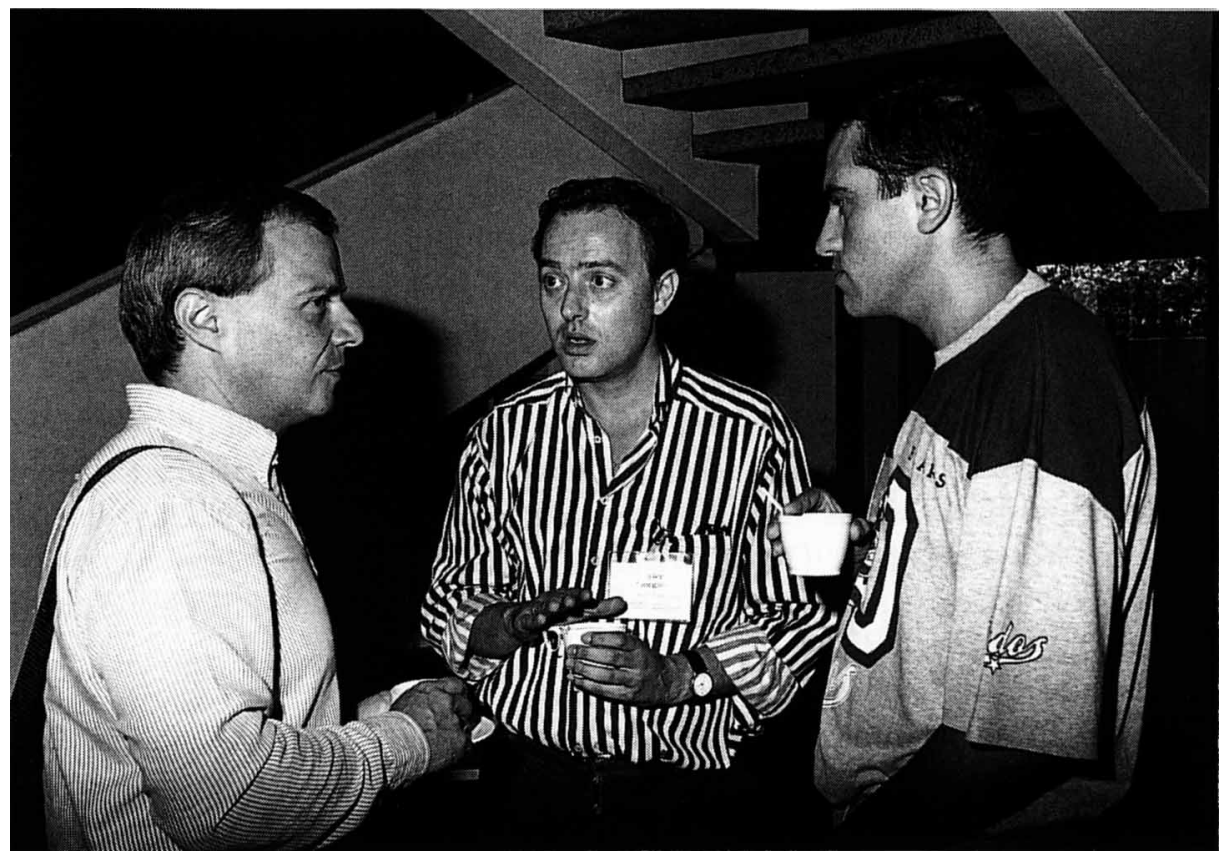

Gustavo Bruzual, Javier Gorgas, Nicolas Cardiel 